Review

\title{
Which factors affect the choice of the inhaler in chronic obstructive respiratory diseases?
}

\author{
Nicola Scichilone ${ }^{a,}{ }^{\text {* }}$, Alida Benfante a, Marialuisa Bocchino ${ }^{\mathrm{b}}$, Fulvio Braido ${ }^{\mathrm{c}}$, \\ Pierluigi Paggiaro ${ }^{\mathrm{d}}$, Alberto Papi ${ }^{\mathrm{e}}$, Pierachille Santus ${ }^{\mathrm{f}}$, Alessandro Sanduzzi ${ }^{\mathrm{b}}$ \\ a Dipartimento di Biomedicina e Medicina Specialistica (DIBIMIS), Sezione di Pneumologia, University of Palermo, Italy \\ b Università degli Studi di Napoli "Federico II", Dipartimento di medicina clinica e chirurgia, Napoli, Italy \\ ${ }^{c}$ Allergy and Respiratory Diseases Clinic, DIMI, University of Genoa, IRCS AOU San Martino-IST, Genoa, Italy \\ ${ }^{\mathrm{d}}$ Cardio-Thoracic and Vascular Department, University Hospital of Pisa, Italy \\ e Respiratory Medicine, Department of Medical Sciences, University of Ferrara, Ferrara, Italy \\ ${ }^{\mathrm{f}}$ Dipartimento di Scienze della Salute, Pneumologia Riabilitativa Fondazione Salvatore Maugeri, Istituto Scientifico di Milano-IRCCS, \\ Università degli Studi di Milano, Italy
}

\section{A R T I C L E I N F O}

\section{Article history:}

Received 14 November 2014

Received in revised form

13 February 2015

Accepted 14 February 2015

Available online 24 February 2015

\section{Keywords:}

Inhaler

Asthma

COPD

Adherence

\begin{abstract}
A B S T R A C T
Inhalation is the preferred route of drug administration in chronic respiratory diseases because it optimises delivery of the active compounds to the targeted site and minimises side effects from systemic distribution. The choice of a device should be made after careful evaluation of the patient's clinical condition (degree of airway obstruction, comorbidities), as well as their ability to coordinate the inhalation manoeuvre and to generate sufficient inspiratory flow. These patient factors must be aligned with the specific advantages and limitations of each inhaler when making this important choice. Finally, adherence to treatment is not the responsibility of the patient alone, but should be shared also by clinicians. Clinicians have access to a wide selection of pressurised metered dose inhalers (pMDIs) and dry powder inhalers (DPIs) that can be used effectively when matched to the needs of individual patients; this should be perceived as an opportunity rather than a limitation.
\end{abstract}

(c) 2015 Elsevier Ltd. All rights reserved.

\section{Introduction}

Chronic diseases require regular treatment. This applies to asthma and chronic obstructive pulmonary disease (COPD), the most common chronic respiratory conditions, which are characterised by persistent airway inflammation and bronchial obstruction. Inhalation is the preferred route of administration when treating respiratory obstructive diseases, because it optimises the delivery of active compounds to the targeted site, while minimising side effects. In this context, the inhaler plays a crucial role in the management of chronic respiratory diseases. The choice of the device can be as important as the choice of the drug. In real-life clinical settings, physicians often discuss on the properties of various drugs with their colleagues and patients, in order to agree

\footnotetext{
* Corresponding author. Dipartimento di Biomedicina e Medicina Specialistica (DIBIMIS), University of Palermo, Via Trabucco 180, 90146 Palermo, Italy. Tel.: +39 091 6802655; fax: +39 0916882842.

E-mail address: nicola.scichilone@unipa.it (N. Scichilone).
}

on the best possible choice for the patient, whereas little consideration is given to the properties of different inhalers. We believe that priority should be given to the choice of the appropriate device, based on patient needs and expectations, followed by the choice of the drug, based on the disease and its severity. A consensus statement by the task force of the European Respiratory Society (ERS) and the International Society for Aerosols in Medicine (ISAM) provides clear recommendations for choosing the best aerosol delivery device based on a patient's actuation-inhalation coordination, level of inspiratory flow, and other clinical conditions [1]. For example, some inhalers require strong inspiratory force, which may not be possible in emergency situations or in children and elderly.

Ideally, patients should use one device for all of their inhaled therapies; obviously, this is not always possible. We believe that differences in efficacy among devices become trivial in case of correct inhalation technique, as supported by evidence-based guidelines from the American College of Chest Physicians/American College of Asthma, Allergy, and Immunology [2]. The key issue is patient training and verification of the inhalation technique. The 
issue remains of whether the drug is able to reach the targeted site with each device. The current review article aims at comparing the available inhaler devices to assess their advantages and limitations in chronic obstructive diseases. The role of the devices with regard to adherence to treatment will be also addressed.

\section{Role of the inhaler in the management of chronic obstructive respiratory diseases}

The goal of therapy is to obtain optimal control of symptoms and, ideally, to alter the natural course of the disease by delivering the correct drug dosage to the site of structural and functional alterations. To achieve this, two conditions must be met: 1) the aerosol formulation must deposit along the bronchial tree, and 2) drug deposition must provide functional and clinical benefits. Both conditions are closely related to the type of device used. Treatment effectiveness is also determined by adherence to therapy. In this context, adherence is influenced by many factors, and inhaler device preference could be one of them. Individuals suffering from chronic respiratory diseases ask for a device that is simple to use, easy to carry and to check, and accomplishing these conditions may help to reduce some of the issues associated with the lack of adherence. Taken together, these observations emphasize the important role played by the inhalation device in clinical practice; choosing the correct inhaler is the primary step for optimal control of the disease.

\section{Key points in managing treatment by inhaled medications}

Despite the clinical importance of inhaled therapy, current guidelines seem to lack a consensus on recommendations for devices. Less than $3 \%$ of the official documents for management of asthma and COPD focus on device-related issues. Guidelines are generally vague and not always evidence-based regarding criteria for choosing inhaler devices, especially for adults, thus allowing clinically irrelevant factors to influence the choice.

Looking at a pressurised metered dose inhaler (pMDI), for instance, the deposition pattern of the inhaled drug is the result of a complex interaction between the device, the aerosol formulation and the patient's inhalation technique. When an aerosol is inhaled, the drug is deposited at the target site, the bronchi, and also unavoidably in the mouth. A variety of inhalation devices have been introduced in clinical practice over time, differing in terms of design (required inspiratory flow rate, actuation), composition (propellant characteristics, carrier substances), dose per inhalation and cost.

Deposition in the bronchial tree occurs through three different modalities: a) inertial impaction, b) sedimentation, and c) diffusion. Inertial impaction occurs when larger particles deposit in proximal airways, where the velocity of airflow is highest. Sedimentation occurs when suspended particles deposit through the force of gravity; these suspended particles may be exhaled before they deposit. The smallest particles are able to reach the distal parts of the lung, where they contact the airway walls because of Brownian motion. Thus, the choice of a device should be driven by the targeted airway site. For example, devices that produce 3-5 $\mu \mathrm{m}$ droplets are used to deliver antibiotics to the proximal airways [3]; on the other hand, smaller particles are needed to reach the distal lung, where most functional and structural airway changes occur in chronic obstructive diseases. Finally, the clinical benefit provided by inhaled therapy is influenced by patient factors, such as adherence, particularly in paediatric and elderly patients. Adherence is critical for the clinical outcomes, as well as correct technique, and this has driven demand for more user-friendly devices. Treatment effectiveness is strongly dependent on the willingness and ability of the patient to perform the prescribed therapy, particularly for chronic or long-term therapies. Training and practice are required to improve delivery of the inhaled drug to the targeted site. Unfortunately, this aspect is often neglected in daily practice and can negatively influence outcomes.

\section{Drug deposition according to device selection}

pMDIs are the most widely used devices. Their correct use requires coordination between inhalation and device actuation. Incorrect technique can limit the effectiveness in daily clinical practice; lack of coordination can occur with pMDIs and represents almost half of all errors [4]. This can lead to ineffective drug delivery to the lungs with excess deposition in the oropharynx. To overcome this limitation, the use of a spacer with pMDIs is often recommended, especially for patients with known or suspected poor coordination (e.g., children, elderly).

The pMDI canister is formulated as either a suspension or a solution, which differ in terms of particle size, plume velocity and duration. In suspension formulations, the active drug is not soluble in the propellant and remains a solid powder in the container. Consequently, the inhaler must be shaken to ensure uniform distribution of the particles and a constant emitted dose at each use. Up to $25 \%$ of patients do not shake the device properly [5], which results in a variable amount of emitted drug for each aerosol puff. Moreover, suspension formulations require a larger orifice to avoid blockage, which in turn induces aerosol plumes of higher velocity and lower duration. Transition from chlorofluorocarbon (CFC) to hydrofluoroalkane (HFA) propellants has allowed a shift from suspension to solution formulations, in which the drug is uniformly distributed.

Breath-activated pMDI (BA-pMDI) devices are also available, releasing drug when inhalation triggers the metered-dose inhaler, avoiding any coordination issues. Currently, there is only one commercially available soft mist inhaler: the Respimat, which atomises the drug solution using mechanical energy imparted by a spring, producing a fine, slow-moving mist, with less deposition in the mouth and throat and relatively higher lung deposition [6]. The Respimat inhaler was designed to aerosolize most of the metered volume in the form of droplets with a diameter of $>1 \mu \mathrm{m}$ (to avoid loss of small droplets during the subsequent exhalation) and $<5.8 \mu \mathrm{m}$ (to facilitate efficient lung deposition). The fine particle fraction (FPF) for the Respimat is approximately 75\% with most formulations. A second parameter that influences the deposition of the drug is the velocity of the particles during inhalation; higher velocities promote particle impaction in the throat. The spray duration of the Respimat is approximately $1.2 \mathrm{~s}$ and is considerably longer than for pMDIs (typically $0.15-0.36 \mathrm{~s}$ ). The long duration of the soft mist allows for better chances of coordinating the inhalation manoeuvre with the drug release [6].

Soon after the introduction of pMDIs in the management of respiratory diseases, dry powder inhalers (DPIs) were developed to deliver therapy in patients with asthma or COPD. Three types of DPIs are available with different handling instructions: single dose (Breezhaler, HandiHaler, Aerolizer), multiple dose (Diskus) and reservoir (Turbuhaler). The major advantage of DPIs is that they do not require coordinated activation, because the drug is not driven by a propellant but is delivered by the inhalation itself. Thus, DPI devices like the Diskus and the Turbuhaler require higher inspiratory flows than pMDIs to assure optimal drug delivery, because both particle size and the amount of drug reaching the lower airways depend on the peak inspiratory flow [7]. In addition, whereas all pMDIs employ the same inhalation technique, each DPI device requires specific training, which may generate confusion among patients, and may increase the risk of errors. 


\section{Comparing devices for chronic obstructive pulmonary diseases}

The first area to explore when comparing different inhaler devices is the particle size range of the emitted dose. Aerosol particle/ droplet size is one of the most important factors influencing the deposition of medications in the airways. A particle size of $2-5 \mu \mathrm{m}$ has the greatest potential to be deposited throughout the bronchial tree. Indeed, smaller particles deposit into the alveoli, where there is no smooth muscle and where systemic absorption is increased [8]. Particles $>5 \mu \mathrm{m}$ tend to settle in the mouth and oesophageal region, where they produce no clinical effect but can potentially produce both local and systemic side effects after gastrointestinal absorption [8]. Consequently, differences in particle size of the aerosol emitted from inhalation devices may influence functional and clinical responses. Aerosol particle size depends on drug-, formulation- and device-related factors. Two parameters are used to define the particle size: the mass median aerodynamic diameter (MMAD), which is the droplet size at which half of the mass of the aerosol is contained in smaller droplets and half in larger droplets, and the fine particle fraction (FPF), meaning the percentage of particles $<5 \mu \mathrm{m}$ in diameter. Generally, FPF is proportional to the fraction of emitted dose reaching the lung. However, it should be noticed that these are extrapolations of in vitro measurements, and should be used with caution in humans. Indeed, lung deposition of the drug depends on several factors that interact, such as the inhalation flow, the particle size and the drug formulation (Fig. 1). Development of environment-friendly HFA propellants has led to the production of extrafine formulations. The Modulite technology has been developed to provide a slow moving cloud of particles with a specific particle size. This was achieved by incorporating a non-volatile component in the formulation and modifying the geometry of the actuator. Currently, there are several single-agent extrafine formulations on the market (beclomethasone, ciclesonide, formoterol), and only one combination product available (beclomethasone/formoterol) [1]. Extrafine solution pMDIs can deliver compounds with a smaller MMAD than other available devices [9]. It is important to note that non-extrafine combinations are also capable of reaching the lung periphery: budesonide/formoterol delivered by a Turbuhaler significantly improved small airway impairment in asthma patients compared with fluticasone/ salmeterol by Diskus [10]. However, peripheral deposition of extrafine combinations is several fold higher compared with nonextrafine combinations [11].

An in vitro study comparing the proportion of the nominal dose of different corticosteroids delivered as fine drug particles from four different DPIs at different flow rates showed that the Turbuhaler device produced a higher proportion of respirable particles

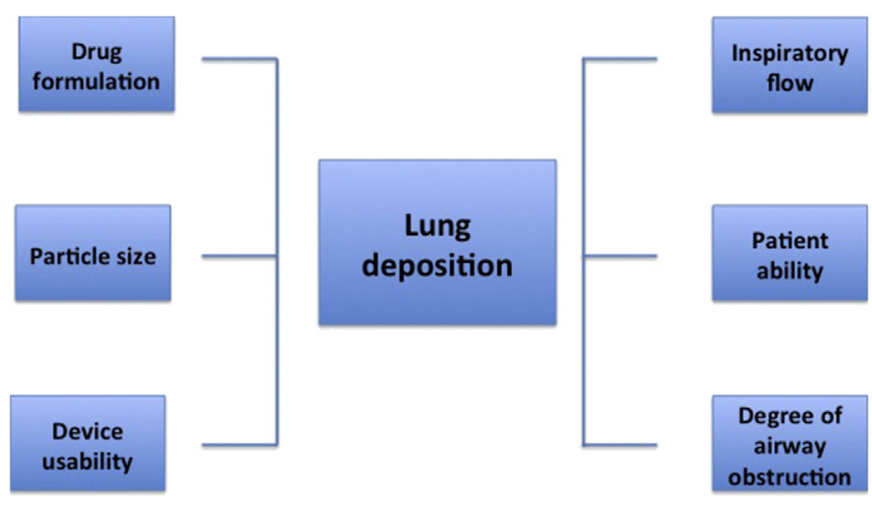

Fig. 1. Description of factors affecting lung deposition of the inhaled drug. than the other devices, and this was consistent across the range of applied inspiratory force [12]. These in vitro findings need to be confirmed with in vivo deposition studies.

Gamma scintigraphy of inhaled radiolabeled drugs shows that the amount of drug deposited in the lungs is a function of the inhalation technique, the inhaled product, or both. This in vivo method revealed significant differences between devices with respect to pulmonary deposition, with values ranging from approximately $5 \%$ to over $50 \%$ for currently available pMDIs and DPIs. However, the relationship between the drug particle size, the deposition of the drug and the clinical and functional benefit is not straightforward. In particular, it is still unknown what is the optimal particle size able to provide greater improvements in lung function. In this regard, the discrepancy between the study from Zanen et al. [13] and that from Usmani et al. [14] which specifically addressed the clinical benefit as a function of the particle size of the inhaled bronchodilator, could be only apparent, since the asthmatic volunteers differed in terms of magnitude of airway obstruction. Findings with this methodology generally replicate those obtained with the Andersen impactor device. Regarding DPIs, Turbuhaler delivers approximately $25-35 \%$ of the labelled dose to the lung, whereas the Diskus delivers about half this amount [15]. The lung deposition from Turbuhaler is significantly greater than from a pMDI [16]. The two-fold improvement in pulmonary deposition with Turbuhaler, compared with Diskus, is consistent with in vitro particle assessment using an Andersen impactor. However, as already mentioned the in vitro findings can be poor predictors of in vivo conditions. The differences in lung deposition may be due to the larger amount of fine particles generated by Turbuhaler, compared with Diskus or the pMDIs, due to disaggregation of the agglomerated formulation in the spiral channels of Turbuhaler. Diskus has coarse particles combined with a lactose carrier that has limited capacity for generating fine particles [17]. Lung deposition is more consistent with Turbuhaler than with Diskus (coefficient of variation $20 \%$ vs. $40 \%$ ) $[15,18]$. Other inhalers, such as NEXThaler, have demonstrated good performance despite the degree of bronchococonstriction and underlying disease. In healthy volunteers, $55 \%$ of the labelled dose was delivered, compared with $56 \%$ in patients with asthma and 55\% in patients with COPD [19]. Breezhaler was studied by Choltorpe and colleagues [20], showing that the medium fine particles fraction was $42.6 \%$ of the labelled dose, with a MMAD of $2.8 \mu \mathrm{m}$ for glycopyrronium. The intrathoracic deposition was of $39 \%$ for glycopyrronium while extrathoracic deposition was about $45 \%$ [20].

Whereas these physical and mechanical characteristics per se do not imply a clinical advantage, it is logical to assume that the clinical benefit is related to the fraction and variability of lung deposition of the active drug. The inspiratory flow must also be considered, because inhaler efficiency is strongly dependent on the production of an adequate peak inspiratory flow. Each inhaler has a minimum energy (i.e., inhalation flow) required to provide efficient disaggregation of the formulation. In the study by Tarsin [7], both Diskus and Turbuhaler seem to require high inspiratory flows to assure optimal drug delivery, and there is a more significant effect of inspiratory flow on variable dosage emission when using the Turbuhaler compared with the Diskus. Whereas minimum inhalation flows are not clearly defined for each device, they are important because of the potential risk for a patient to receive a non-appropriate dose. In general, very young and elderly patients, and those experiencing a severe exacerbation may not be able to generate sufficient inhalation flow to generate particles capable of reaching the lungs. In a paper by Pavkov the peak inspiratory flow through Breezhaler was evaluated in 26 moderate to severe COPD patients, who were able to generate a flow greater than $60 \mathrm{~L} / \mathrm{min}$ [21]. Very few data are available regarding lung deposition: it was 
estimated that about $34 \%$ of the labelled dose of indacaterol could be delivered through Breezhaler [22].

Dewar demonstrated that patients with severe bronchial obstruction from COPD can generate sufficient peak inspiratory flow to operate Turbuhaler effectively [23]. Due to the large amount of small particles and the moderate inbuilt resistance in Turbuhaler, which opens up the vocal cords during inhalation, Turbuhaler is associated with higher lung deposition $(25-40 \%$ of the delivered dose) compared with pMDIs and other DPIs $[12,16]$. A good correlation has been found between lung deposition and clinical efficacy. A high lung deposition always results in the best ratio between clinical efficacy and risk of unwanted systemic activity [24]. Studies with Turbuhaler also show that the in vivo variation in lung deposition is significantly lower compared with a pMDI or, for example, the Diskus inhaler, and much lower than the in vitro dose variability seen in laboratory tests [18].

However, due to the complex interaction of flow rate, particle sizes, acceleration rates/disaggregation and inhalation volumes, many questions remain to be fully answered. Clinical randomized trials do not reflect the population of patients using inhalers. Future studies that can assess all of these separate parameters in a variety of patients, in real-life, would provide beneficial insight into this area. Other parameters, such as the feedback system or the required steps to activate the device variably affect the effectiveness of the inhaled treatment in clinical settings. Table 1 summarizes the main properties for each inhaler.

\section{Patient adherence in the management of chronic obstructive respiratory diseases}

Patients play a major role in determining the success or failure of treatment. "Adherence" must be distinguished from "compliance", the difference being in the patient's willingness to accept therapy [25]: the "non-compliant" patient simply ignores prescriptions. Adherent patients take medications as prescribed, whereas "nonadherent" patients fail to do so despite their willingness and acceptance of therapy. The unwitting non-adherence that occurs when a patient does not know the proper inhaler technique or does not understand the difference between a rescue and controller medication is common in real life. The issue of patient age is particularly important in unintentional non-adherence. Young children dependent on others for delivery of medication and are frequently unable to communicate their perception of symptom severity. In these patients, the common use of nebulizers makes the administration of asthma medication particularly tedious and timeconsuming [26]. However, other factors, including fear of stigmatisation at school, fear of side effects, fear of dependence can significantly reduce adherence in school-age children [27]. As psychological distress is associated with non-adherence, accurate screening for unhealthy behaviours should be part of a comprehensive approach to adolescents with asthma.

In elderly patients, unintentional non-adherence to inhalation therapy represents a complex problem that may lead to significant impairment of symptom control. Elderly patients often suffer from cognitive impairments, hearing or visual problems or other

Table 1

Main features for each device.

\begin{tabular}{llll}
\hline Inhaler & Loaded & Feedbacks & Dose counter \\
\hline Breezhaler & NO & NO & NO \\
Handihaler & NO & NO & NO \\
Respimat & NO & NO & NO \\
Diskus & YES & NO & YES \\
Turbuhaler & YES & NO & YES \\
Nexthaler & YES & NO & YES \\
\hline
\end{tabular}

physical disabilities (e.g., arthritis, tremors and poor coordination) that significantly affect their ability to understand and follow treatment regimens. In addition, elderly patients often have multiple chronic diseases requiring multiple medications, and treatment complexity is a major risk factor for reduced adherence. As previously discussed, the availability of a multitude of inhaler devices can be confusing to the patient. Switching between different inhalers negatively affects care, as inhaler classes and brands differ in design, and each device has its own unique requirements and inhalation technique [28].

Patient training is critical for the correct use of inhaler devices and the effectiveness of therapy. Healthcare providers should not assume that correct inhaler use is intuitive, or obvious from the printed instructions provided with the inhaler. Moreover, they should not rely on verbal communication alone, but rather provide clear visual "step by step" instructions with pictures and encourage patients to take notes on the instruction sheet. Unfortunately, both patients and healthcare providers underestimate the issue of inhalation device incompetence. The fault may also occur in healthcare provider if competence or training skills are less than optimal [29].

Patient education is critical for reducing unwitting non-adherence, and thereby increasing the overall effectiveness of inhalation therapy. Education entails simplification, demonstration and repetition, and should include an understanding of why the patient needs the inhaler, how the inhaler works, and the steps required to use it correctly. International guidelines recommend that training should be provided at each visit, and that patients should be encouraged to bring all of their inhalers to each visit and demonstrate their inhalation technique; however, a consensus is lacking on how to match patient incompetence with criteria for selecting an alternative inhaler device. We suggest that rather than insisting with training a patient with a specific inhaler device, it would be more appropriate to match the device with the behaviour and the skills of the patient. The "idealhaler" does not exist in real life. Instead, healthcare providers should carefully evaluate which device would fit the needs and ability of the patient. Importantly, the patient's preference should not be neglected.

\section{Conclusions}

Meta-analyses and systematic reviews $[2,3,30]$ indicate that all inhalers can be effective and can achieve a similar therapeutic effect when patients use the inhalation technique recommended by the manufacturer, although different doses may be required. These observations are often documented in randomised controlled trials, where patients receive more inhaler-technique training and counselling on the importance of adherence than do patients in routine clinical practice. Real-life investigations confirm that most patients make at least one error when using the inhaler, and this often affects delivery of the drug to the bronchial tree. Thus, the responsibility for maintaining adherence to treatment is shared by the patient and the clinician, who must provide adequate training and monitoring. Clinicians have a plethora of effective devices that can be applied to specific patient needs; this should be perceived as an opportunity rather than a limitation.

\section{Acknowledgements}

English language editing and styling assistance was provided by Edra LSWR, Elsevier, and funded by AstraZeneca.

\section{References}

[1] Laube BL, Janssens HM, de Jongh FH, Devadason SG, Dhand R, Diot P, et al What the pulmonary specialist should know about the new inhalation therapies. Eur Respir J 2011;37(6):1308-31. 
[2] Dolovich MB, Ahrens RC, Hess DR, Anderson P, Dhand R, Rau JL, et al. Device selection and outcomes of aerosol therapy: evidence-based guidelines: American College of Chest Physicians/American College of Asthma, Allergy, and Immunology. Chest 2005;127(1):335-71.

[3] Rubin BK. Pediatric aerosol therapy: new devices and new drugs. Respir Care 2011 Sep;56(9):1411-21.

[4] Giraud V, Roche N. Misuse of corticosteroid metered-dose inhaler is associated with decreased asthma stability. Eur Respir J 2002;19(2):246-51.

[5] van der Palen J, Klein JJ, van Herwaarden CL, Zielhuis GA, Seydel ER. Multiple inhalers confuse asthma patients. Eur Respir J 1999;14(5):1034-7.

[6] Dalby RN, Eicher J, Zierenberg B. Development of respimat soft mist inhaler and its clinical utility in respiratory disorders. Med Devices Auckl 2011;4: $145-55$.

[7] Tarsin WY, Pearson SB, Assi KH, Chrystyn H. Emitted dose estimates from Seretide Diskus and Symbicort Turbuhaler following inhalation by severe asthmatics. Int J Pharm 2006;316(1-2):131-7.

[8] Chrystyn H. Anatomy and physiology in delivery: can we define our targets? Alergy 1999;54(Suppl. 49):82-7.

[9] Fabbri LM, Nicolini G, Olivieri D, Papi A. Inhaled beclometasone dipropionate/formoterol extra-fine fixed combination in the treatment of asthma: evidence and future perspectives. Expert Opin Pharmacother 2008;9(3): 479-90.

[10] Hozawa S, Terada M, Hozawa M. Comparison of budesonide/formoterol Turbuhaler with fluticasone/salmeterol Diskus for treatment effects on small airway impairment and airway inflammation in patients with asthma. Pulm Pharmacol Ther 2011;24(5):571-6.

[11] Leach CL, Kuehl PJ, Chand R, Ketai L, Norenberg JP, McDonald JD. Characterization of respiratory deposition of fluticasone-salmeterol hydrofluoroalkane134a and hydrofluoroalkane-134a beclomethasone in asthmatic patients. Ann Allergy Asthma Immunol 2012:108(3):195-200.

[12] Olsson B. Aerosol particle generation from dry powder inhalers: can they equal pressurized metered dose inhalers? J Aerosol Med 1995;8(Suppl. 3): S13-8.

[13] Zanen P, Go LT, Lammers JW. Optimal particle size for $\beta 2$-agonists and anticholinergic aerosols in patients with severe airflow obstruction. Thorax 1996;51:977-80.

[14] Usmani O, Biddiscombe MF, Nightingale JA, Underwood SR, Barnes PJ. Effects of bronchodilator particle size in asthmatic patients using monodisperse aerosols. J Appl Physiol 2003;95:2106-12.

[15] Thorsson L, Edsbäcker S, Källén A, Löfdahl CG. Pharmacokinetics and systemic activity of fluticasone via Diskus and pMDI, and of budesonide via Turbuhaler Br J Clin Pharmacol 2001;52(5):529-38.
[16] Thorsson L, Kenyon CJ, Newman SP, Borgström L. Lung deposition of budesonide in asthmatics: a comparison of different formulations. Int J Pharm 1998;168(1):119-27.

[17] Borgström L, Asking L, Thorsson L. Idealhalers or realhalers? A comparison of Diskus and Turbuhaler. Int J Clin Pract 2005 Dec;59(12):1488-95.

[18] Agertoft L, Pedersen S. Lung deposition and systemic availability of fluticasone Diskus and budesonide Turbuhaler in children. Am J Respir Crit Care Med 2003;168(7):779-82.

[19] Corradi M, Chrystyn H, Cosio BG, Pirozynski M, Loukides S, Louis R, et al NEXThaler, an innovative dry powder inhaler delivering an extrafine fixed combination of beclometasone and formoterol to treat large and small airways in asthma. Expert Opin Drug Deliv 2014 Sep;11(9):1497-506.

[20] Colthorpe P, Voshaar T, Kieckbusch T, Cuoghi E, Jauernig J. Delivery characteristics of a low-resistance dry-powder inhaler used to deliver the longacting muscarinic antagonist glycopyrronium. J Drug Assess 2013;2:11-6.

[21] Pavkov R, Mueller S, Fiebich K, Singh D, Stowasser F, Pignatelli G, et al Characteristics of a capsule based dry powder inhaler for the delivery of indacaterol. Curr Med Res Opin 2010 Nov;26(11):2527-33.

[22] Molimard M, D'Andrea P. Once-daily glycopyrronium via the Breezhaler ${ }^{\mathbb{R}}$ device for the treatment of COPD: pharmacological and clinical profile, vol. 5; 2013. p. 503-17.

[23] Dewar MH, Jamieson A, McLean A, Crompton GK. Peak inspiratory flow through Turbuhaler in chronic obstructive airways disease. Respir Med 1999 May;93(5):342-4.

[24] Borgstrom L, Derom E, Stahl E, Wahlin-Boll E, Pauwels R. The inhalation device influences lung deposition and bronchodilating effect of terbutaline. Am J Respir Crit Care Med 1996;153:1636-40.

[25] Osterberg L, Blaschke T. Adherence to medication. N Engl J Med 2005 Aug 4;353(5):487-97.

[26] Graves MM, Adams CD, Portnoy JM. Adherence in young children with asthma. Curr Opin Allergy Clin Immunol 2006;6(2):124-7.

[27] Bender BG, Bender SE. Patient-identified barriers to asthma treatment adherence: responses to interviews, focus groups, and questionnaires. Immunol Allergy Clin N Am 2005;25(1):107-30.

[28] Thomas M, Price D, Chrystyn H, Lloyd A, Williams AE, von Ziegenweidt J. Inhaled corticosteroids for asthma: impact of practice level device switching on asthma control. BMC Pulm Med 2009;9:1.

[29] Hanania NA, Wittman R, Kesten S, Chapman KR. Medical personnel's knowledge of and ability to use inhaling devices. Metered-dose inhalers, spacing chambers, and breath-actuated dry powder inhalers. Chest 1994;105(1):111-6.

[30] Hess DR. Aerosol delivery devices in the treatment of asthma. Respir Care 2008;53(6):699-723. 